\title{
Carnets
}

Revue électronique d'études françaises de l'APEF

Deuxième série - 3 | 2015

Insulaire

\section{Un rêve en haute mer ou la vérité trompeuse de Supervielle}

\section{Maria Eugénia Pereira}

\section{(2) OpenEdition}

Journals

Édition électronique

URL : http://journals.openedition.org/carnets/1404

DOI : 10.4000/carnets. 1404

ISSN : 1646-7698

Éditeur

APEF

Référence électronique

Maria Eugénia Pereira, « Un rêve en haute mer ou la vérité trompeuse de Supervielle », Carnets [En ligne], Deuxième série - 3 | 2015, mis en ligne le 28 février 2015, consulté le 01 mai 2019. URL : http:// journals.openedition.org/carnets/1404; DOI : 10.4000/carnets.1404

Ce document a été généré automatiquement le 1 mai 2019.

\section{(c) (i) (8)}

Carnets est mis à disposition selon les termes de la licence Creative Commons - Atribution - Pas d'utilisation commerciale 4.0 International. 


\title{
Un rêve en haute mer ou la vérité trompeuse de Supervielle
}

\author{
Maria Eugénia Pereira
}

1 Selon Supervielle, nos sens, parce qu'ils sont défaillants, ne nous permettent pas de saisir toute la réalité de l'univers - nous devons consentir que notre raison, sans doute beaucoup trop restrictive, et que notre mémoire, beaucoup trop limitée, aident peu à la découverte d'une surnature, si nous n'y ajoutons pas la sensibilité. En somme, par l'imagination, cet auteur ne cherche pas véritablement à atteindre l'inaccoutumé ou à dire l'indécis, il préfère soulever le doute, dire des interrogations et susciter le mystère. Tout comme nous le dit Gaston Bachelard, l'imagination «est d'abord un facteur d'imprudence qui nous détache des lourdes stabilités. (...) [C]ertaines rêveries poétiques sont des hypothèses de vies qui élargissent notre vie en nous mettant en confiance dans l'univers » (Bachelard, 1960 : 7).

2 Supervielle possède une capacité singulière d'introduire l'arbitraire dans le non arbitraire, de dépasser les bornes du visible et du concret. Or, en tant qu'auteur de récits brefs, il subit, dès lors, une forte entrave : il ne peut jouer sur la longueur du récit pour exposer les règles des mondes imaginaires et pour les rendre crédibles, pour faire grandir son lecteur, pour faire grossir ses compétences de lecture. L'écrivain doit économiser ses mots, mais être tout à la fois suffisamment convainquant pour que l'attachement du lecteur à l'héroïne et à l'histoire soit rapide et pour que celui-ci laisse aller son imagination. Comme l'affirme Daniel Grojnowski, «le récit bref forme à lui-même un univers clos, autonome, un microcosme événementiel» (Grojnowski, 2000: 37), aussi l'auteur doit-il, avant tout, faciliter l'entrée en fiction:

Comment s'était formée cette rue flottante? Quels marins, avec l'aide de quels architectes, l'avaient construite dans le haut Atlantique à la surface de la mer, audessus d'un gouffre de six mille mètres? Cette longue rue aux maisons de briques rouges si décolorées qu'elles prenaient une teinte gris-de-France, ces toits d'ardoise, de tuile, ces humbles boutiques immuables? Et ce clocher très ajouré ? Et ceci qui ne contenait que de l'eau marine et voulait sans doute être un jardin clos de murs, garnis de tessons de bouteilles, par-dessus lesquels sautait parfois un poisson?

Comment cela tenait-il debout sans même être balloté par les vagues? 
Et cette enfant de douze ans si seule qui passait en sabots d'un pas sûr dans la rue liquide, comme si elle marchait sur la terre ferme ? Comment se faisait-il...?

Nous dirons les choses au fur et à mesure que nous les verrons et que nous saurons. Et ce qui doit rester obscur le sera malgré tout (Supervielle, $1958: 10$ ).

Dans cet incipit du conte "L'Enfant de la haute mer", interrogations et points de suspension installent le suspense et préparent le mystère ; par la suite, un «nous » met à l'épreuve le pacte de lecture et l'autorité narrative et rend la narration indécidable; et, alors que le doute, le silence et l'indécidabilité avaient commencé à rendre la narration floue, le commentaire du narrateur vient clairement instaurer l'ambigüité : « Nous dirons les choses au fur et à mesure que nous les verrons et que nous saurons. Et ce qui doit rester obscur le restera malgré tout ». La curiosité du lecteur a donc été vivement suscitée et, désormais, il va avoir envie d'accompagner le reste du récit.

4 L'intrusion de l'insolite est passée par la voix même du narrateur qui ne cherche pas à faire la part des choses et nous présente le surnaturel de façon aussi naturelle que le réel :

À l'approche d'un navire, avant même qu'il fût perceptible à l'horizon, l'enfant était prise d'un grand sommeil, et le village disparaissait, complètement sous les flots. Et c'est ainsi que nul marin, même au bout d'une longue-vue, n'avait jamais aperçu le village ni même soupçonné son existence (Supervielle, 1958 : 9-10).

Le lecteur est désormais entré dans un univers ambivalent, entre réalité et sur-réalité, et se laisse envouter par la voix du narrateur, qui adopte le point de vue du seul personnage du conte pour nous faire partager ses pensées, ses sensations et ses émotions et, ainsi, résoudre l'antinomie du monde fictionnel.

Nous suivons donc, sans le savoir encore, la voix d'un fantôme - l'Enfant de la haute mer -, mais nous avons déjà pris conscience du fait que nous sommes en présence d'un être singulier et, qu'en pénétrant dans son univers, il nous faut accepter que l'inusuel, l'insolite font partie du réel. Nous assumons dès lors que nous nous trouvons dans un monde vacillant, tout à la fois proche et lointain du nôtre, et que nous évoluons dans l'histoire au gré du narrateur et en redéfinissant les frontières entre le réel et le surnaturel.

7 Dans «L'Enfant de la haute mer ", la "rue flottante ", la "ville flottante » ou, plus précisément, l'île-flottante, est un lieu où le regard se perd, puisqu'aucun point fixe n'aide à orienter et à organiser la vision. L'espace construit est, néanmoins, porteur d'un repère réel qui assure la matérialité des lieux: l'«Atlantique» (Supervielle, 1958: 9). Hormis cette indication, qui semble vouloir assurer la matérialité du lieu, le récit est dépouillé de ses déterminations spatiales ordinaires, tout autre point de repère en ayant été aboli, et, par conséquent, cet élément aquatique constitue une sorte de néant. En effet, cette localisation spatiale semble vouloir jouer sur la détermination et la précision, mais le lecteur plonge progressivement dans le vide, dans l'imprécision et l'indétermination: «Mais l'océan même, celui qu'elle voyait sur les cartes, elle ne savait pas se trouver dessus, bien qu'elle l'eût pensé un jour, une seconde » (Supervielle, $1958: 13$ ). Un ailleurs surgit, à la frontière du palpable, proche de la réalité.

8 Par conséquent, le nom, "Atlantique », paraît vouloir garantir la présence effective du lieu et sa localisation géographique, mais il n'est, en fait, dans le récit, que le reflet inversé de la réalité ici-bas et devient présence incertaine, déracinée de la réalité. Paroxystiquement démesuré, l'espace réel acquiert une dimension surnaturelle, qui déracine le lecteur. Gaston Bachelard explique que « [1]'immensité est, pourrait-on dire, une catégorie philosophique de la rêverie. (...) la contemplation de la grandeur détermine 
une attitude si spéciale, un état d'âme si particulier que la rêverie met le rêveur en dehors du monde prochain, devant un monde qui porte le signe d'un infini » (Bachelard, 1978 : 169). L'objet proche, l'Atlantique, devient, par la rêverie, l'espace d'un ailleurs. La mer de Supervielle est donc la sœur jumelle de celle se trouvant sur la Terre, mais l'écrivain, par son pouvoir poétique, dématérialise ce que le lecteur connaît et lui ôte son «illusion de percevoir le monde dans la plénitude de son existence » (Dewulf, 2000, TI : 55). En effet, la mer devient un lieu fictif où se reflète l'irréalité du monde.

L'espace superviellien est donc étrange, puisqu'il nous situe de l'autre côté de l'ici-bas ; il nous place dans le méconnu, mais tout en même temps dans le proche, dans le familier. Le personnage se situe, en fait, à la frontière de la vie et de la mort : "cette enfant de l'Océan, [est] née un jour du cerveau de Charles Liévens, de Steenvoorde, matelot de pont du quatre-mâts Le Hardi, qui avait perdu sa fille âgée de douze ans, pendant un de ses voyages » (Supervielle, $1958: 18$ ).

Georges Poulet considère que l'Océan, chez Supervielle, est

[une] place vide, [un] espace intérieur apparu dans la pensée du poète à la suite du retrait des souvenirs. L'espace c'est l'oubli. Et puisque l'oubli est la disparition du passé, c'est-à-dire de la vie antérieure, tant dans son mouvement progressif que dans son contenu sensible, l'espace (comme la mort) est cette lacune immense que cause dans l'âme le remplacement du temps vécu par un temps vide (Poulet, 1964 : 120).

11 Mais l'océan, en tant que porteur de souvenirs, "n'en est pas moins aussi le milieu translateur, grâce auquel ce qui est perdu se retrouve » (Poulet, 1964: 124); grâce à la mémoire " ce qui avait été laissé en arrière vient rejoindre en avant un cher devancier " (Poulet, 1964 : 124).

Supervielle nous fait donc prendre contact avec des lieux intermédiaires, « intersidéraux » (cf. Dewulf, 2000, TI : 55), imprégnés de la présence de la mort :

L'espace où se meut celui-ci est peuplé de figures incertaines et impalpables dont on ne sait si elles représentent des morts persistant à marquer le monde de leur empreinte, si légère soit-elle, ou des vivants que voile une absence fondamentale, qu'elle soit d'ordre physique ou mental (Dewulf, 2000, TI : 56).

13 L'espace de Supervielle est constitué de deux univers, parallèles et en même temps étrangers l'un à l'autre, qui se superposent en miroir et qui sont liés par une parenté mystérieuse: l'écriture. Soumis à de profonds changements par la main de l'artiste, l'univers réel acquiert la dimension du rêve et brouille le lecteur. Le passage du réel à l'irréel correspond au passage de la vie à la mort et acquiert la dimension de l'infini. Gaston Bachelard nous dit que, dans "notre âme nocturne", «la mort [est] conçue comme un départ sur l'eau » (Bachelard, 1942 : 103).

Profondément ingénieux, Supervielle a su situer son récit dans un lieu capable de traduire métaphoriquement sa spécificité artistique ; "Être dans un endroit dont on ne sait rien », nous dit Georges Poulet, "c'est être dans un lieu aussi anonyme qu'on l'est soi-même, c'est multiplier la conscience cruelle de sa propre étrangeté par la conscience de l'étrangeté du monde » (Poulet, 1964: 111). L'espace superviellien est un des artifices littéraires qui remplit une des plus importantes fonctions au sein de la narration: ses propriétés objectives, «soulignées par le contexte, [lui] confèrent automatiquement la valeur d'une métaphore, avant même que le lecteur ne songe à les interpréter » (Dupuis, 1987 : 229). 

son action dans un milieu temporel comparable au sien, mais celui-ci se rend bien rapidement compte que le temps s'effiloche pour concrétiser la valeur double ou multiple du temps représenté. quotidienne, des événements qui se répètent au jour le jour sur l'île flottante. L'auteur use d'imprécision, lorsqu'il adopte les notations temporelles, dans la mesure où il cherche à rendre compte de «la mécanique [qui compte] les moments de la vie » (Hiddleston, $1965: 144)$ :

Le matin, une demi-livre de pain frais, enveloppé dans du papier, attendait l'enfant sur le comptoir de marbre de la boulangerie (...). Elle était debout de bonne heure (...). Une heure avant le coucher du soleil elle commençait à fermer les volets avec simplicité. (...) Tous les matins elle allait à l'école communale (...) (Supervielle, $1958: 10,11$ et 13).

Le temps s'écoule lentement sur cette île flottante, rythmé par le passage de «la nuit, [du jour], [du] jour, [de la] nuit » (Supervielle, 1958 : 14), et par les répétitions des gestes, des tâches et des paroles de la petite fille. Le temps, d'après James Hiddleston, est « arythmique » (Hiddleston, $1965:$ 144), puisque la jeune fille piétine, n'avance pas dans le temps : « Le temps ne passait pas sur la ville flottante : l'enfant avait toujours douze ans » (Supervielle, 1958 : 15). James A. Hiddleston explique encore que le temps superviellien «ne s'écoule pas vers l'avenir, mais se compose d'une série de moments entrecoupés, saccadés, comme si une divinité malveillante renouvelait à chaque instant le don de la vie, s'interrogeant, hésitant avant d'en accorder un autre» (Hiddleston, 1965: 144). Vouloir suspendre le temps est inutile et toute tentative de rébellion est vaine, le temps reste immuable :

L'enfant, percevant pour la fois un bruit qui lui venait des hommes, se précipita à la fenêtre et cria de toutes ses forces :

«Au secours!»

Et elle lança son tablier d'écolière dans la direction du navire.

L'homme de barre ne tourna même pas la tête. Et un matelot, qui faisait sortir de la fumée de sa bouche, passa sur le pont comme si de rien n'était. (Supervielle, 1958 :

16)

(...) Et la fillette qui n'avait pas une égratignure dut recommencer d'ouvrir et de fermer les volets sans espoir, et de disparaître momentanément dans la mer dès que le mât d'un navire pointait à l'horizon (Supervielle, 1958 : 17).

La temporalité se trouve vidée de sa principale fonction, qui est de déterminer la durée d'une action, d'un épisode, et gère le vide. Cette enfant ne peut aucunement se projeter dans l'avenir, puisque le temps se trouve suspendu, arrêté dans le passé. Le présent n'ayant aucun sens, dès qu'elle se sent exister, ce moment lui est volé et la vie se fige dans l'immobilité qu'est la mort.

ersion de l'écriture superviellienne visant « l'omniprésente menace de l'opacité et de l'incertitude» (Dewulf, 2000, TI : 54), le temps ne peut donc pas être porteur des repères conventionnels - tels que la chronologie et la linéarité -, garants de la réalité des choses. Le statisme du temps de «L'Enfant de la haute mer » rend l'univers insaisissable et le place dans l'éternité.

Dans ce village flottant, l'apparent passage des jours semble vouloir garantir la présence effective du temps connu, mais il n'en est, en fait, qu'un faux reflet puisqu'il se trouve dématérialisé. En effet, la présence de repères temporels ne participe en rien à la 
consistance de l'univers, mais convoque, au contraire, un temps ambigu. C'est par la démesure qu'il rend compte du temps et qu'il gère son univers phantasmatique, constitué d'apparences du monde réel.

21 Le temps est circulaire et Supervielle en est le centre : passé et futur, proche et lointain, long et court n'ont plus la valeur référentielle conventionnelle et se rejoignent, se touchent dans un temps né de sa rêverie. Hiddleston en explique, ainsi, la raison :

La question du temps, et par extension celle de la mort, reste sans solution. Au sommet de la rêverie superviellienne le monde se dispose en cercle autour de son esprit et de son corps. Parlant du centre, l'esprit du poète, s'élargissant en cercles concentriques, domine l'espace, où tout est dorénavant présent et absent en même temps. Les distances ainsi vaincues, des rapports s'établissent entre les êtres autrefois séparés (Hiddleston, $1965: 193$ ).

Le créateur invente une zone spatiale intermédiaire et une dimension temporelle «flottante », afin de donner un sens à l'absurdité de l'existence humaine.

Supervielle profite donc de son pouvoir créateur pour abolir, détenir, inverser, juxtaposer le temps. À la façon de Bachelard, il croit qu'« il n'y a pas de date et de durée où il n'y a pas construction; [qu'] il n'y a pas de date sans dialectique, sans différences » (Bachelard, 1993 : 51).

24 L'Enfant de la haute mer se trouve, ainsi, libérée du joug du temps et elle y évolue au rythme de son imagination. Le temps ne coule pas selon un ordre linéaire et chronologique, mais selon un ordre instauré par l'esprit, c'est pourquoi il est un des éléments qui participe au merveilleux et au magique du récit. Il « affecte chaque aspect de la fiction, la forme et le moyen (le langage). Chaque chose, comme la vie, porte son propre horaire» (Mendilow, 1952: 31). Comme auteur qui s'étonne de tout ce qui l'entoure, Supervielle réhabilite le temps réellement institué d'une poésie qui le rend flou et imprécis.

Dans «L'Enfant de la haute mer ", l'histoire s'engage en conte et nous conduit, dès lors, vers des contrées mystérieuses et lointaines, où la réalité se constitue par un glissement de l'extérieur vers l'intérieur. Il faut donc savoir plonger dans les eaux profondes du discours narratif, découvrir l'insolite et l'accepter comme naturel.

Par conséquent, le lecteur doit accepter le récit tel qu'il est et ne pas essayer de le réduire à l'explicable, ne pas le maintenir à distance par le fait même de son étrangeté : pour entrer dans l'atmosphère du conte, le lecteur doit être capable de faire confiance à l'auteur, de partager avec lui son monde intérieur.

27 Supervielle nous donne sa représentation intérieure du monde, mais avec le souci d'y faire croire : il use le narrateur pour envoûter le lecteur, pour lui faire partager sa pensée. Ce dernier commence par jouer avec le quotidien :

Tous les matins elle allait à l'école communale avec un grand cartable enfermant des cahiers, une grammaire, une arithmétique, une histoire de France, une géographie (Supervielle, $1958: 11$ ).

puis, il installe le lecteur dans le rêve :

À l'approche d'un navire, avant même qu'il fût perceptible à l'horizon, l'enfant était prise d'un grand sommeil, et le village disparaissait, complètement sous les flots. Et c'est ainsi que nul marin, même au bout d'une longue vue, n'avait jamais aperçu le village (Supervielle, $1958: 9-10$ ).

dans le monde de l'enfance : 
Elle n'était pas très jolie à cause de ses dents un peu écartées, de son nez un peu trop retroussé, mais elle avait la peau très blanche avec quelques taches de douceur, je veux dire de rousseur (Supervielle, $1958: 9-10$ ).

dans l'intimité, pour le faire sombrer dans ces eaux mystérieuses :

Et sa petite personne commandée par des yeux gris, modestes mais très lumineux, vous faisait passer dans le corps, jusqu'à l'âme, une grande surprise qui arrivait du fond des temps (Supervielle, $1958: 9-10$ ).

Douce désinvolture et tendre humour : deux procédés qui servent à rappeler au lecteur que les événements sont narrés au gré d'une rêverie métaphysique, qui repose sur la dialectique du réel et de l'irréel : toute la dynamique de l'action peut être expliquée par ce va-et-vient mental entre le quotidien et le surnaturel. Par conséquent, le conte repose sur une équivoque, que la narration n'essaie aucunement de résoudre.

Dans l'univers superviellien nous glissons progressivement, sans que nous nous en apercevions, dans le domaine des fantômes, pour mieux révéler la condition de l'homme et ce qu'elle possède de plus effrayant : la mort. La solitude, le désespoir et l'angoisse sont les sentiments qui envahissent l'homme dans ce monde où règnent le silence, l'absence et la mort. L'Enfant de la haute mer, isolée sur cette île flottante, souffre de vivre seule ; par conséquent, elle symbolise également l'impossibilité de dialoguer avec les autres, de maintenir un contact avec le monde extérieur.

Le problème majeur de ce personnage est de ne pas réussir à sortir de la solitude infernale où il se trouve. Il s'alimente du rêve, mais le désarroi qu'il éprouve de ne point parvenir à s'emparer du monde par le rêve le mène à sombrer dans le néant. En effet, l'Enfant de la haute mer est malheureuse de vivre dans l'irréalité de cette rue flottante, puisqu'elle se trouve privée de l'existence elle-même ; elle voudrait pouvoir s'en libérer et donner une forme concrète à ses propres images intérieures, réaliser ses rêves; or, son état de fantôme rend son désir impossible. En fait, cette fillette se trouve coincée entre le passé et l'avenir, c'est-à-dire, entre l'enfance et l'âge adulte, et le surnaturel - la mort bouscule le réel de façon bouleversante.

Cette enfant souffre donc d'une déchirure intérieure provoquée par la prise de conscience du tragique de son existence, c'est pourquoi, dans la quête d'un absolu humain, elle essaie de s'élever vers un ailleurs. Jetée dans un monde absurde et étrange - où la vie n'a pas de sens apparent -, elle refuse, néanmoins, d'admettre qu'il n'y ait pas de sens caché derrière les apparences, c'est la raison pour laquelle elle se lance dans une quête de soimême.

En fait, l'écrivain ne fait que relâcher la vigilance de la raison et transformer le monde selon son intérieur afin de faire naître une créature qui ne dépendra, désormais, plus que de lui. Cet être fictionnel est extraordinaire parce qu'il est né d'un sentiment ambivalent, d'une relation particulière avec le monde. Claude Roy nous explique que Supervielle « axe sa vision sur cet 'ancrage' parmi les choses que nous réalisons au moyen de notre corps " (Roy, 1970 : 41), c'est-à-dire qu'il appréhende le monde par ses sens et sa perception et, qu'en tant que poète, il est capable d'en déformer les traits à sa guise. Une fois de plus selon Claude Roy, « le sentiment qui lui est le plus naturel, est celui de la simultanéité, la certitude de se mouvoir, d'exister dans un monde sinon fermé, du moins sans coutures » (Roy, 1970: 41), où le dedans et le dehors, le réel et l'imaginaire cohabitent dans une union pacifique.

En tant qu'homme, Supervielle s'étonne de tout ce qui l'entoure et, par conséquent, il fait de son écriture, nous dit Sabine Dewulf, le «miroir enchanté, dont la surface, au lieu de 
réfléchir passivement la réalité instituée, nous invite au contraire à nous défier de sa limpidité. Il nous faut descendre en d'obscures profondeurs, jusqu'à nous rapprocher de 'ce centre mystérieux où bat le cœur même de la poésie'« (Dewulf, 2000, TII : 15).

"L'Enfant de la haute mer", met donc en scène une morte-vivante, une noyée, un fantôme ou, plus vaguement, une ombre. "Chez Supervielle, on glisse ", nous dit Ovid S. Crohmàlniceanu, «sans s'en apercevoir des noyés aux fantômes. Le poète s'imagine la demi-existence de ceux qui ont sombré dans les profondeurs liquides (...). Un endroit où règne un silence total. $\mathrm{Au}$ sein de ce monde, le fantôme apporte l'oubli» (Crohmàlniceanu, 1995 : 86).

Notre premier contact est avec l'étrange : le narrateur nous fait pénétrer dans le domaine du surnaturel, mais il en atténue les effets, en le mitigeant avec le réel, parce qu'il a su anticiper sur les doutes du lecteur et trouver les explications à tant d'événements improbables (cf. Supervielle, 1958: 10). Le lecteur entre dans le domaine du rêve sans aucun soubresaut, sans aucun recul, puisque, par ce procédé, seule sa curiosité a été éveillée. Yves-Alain Favre fait un commentaire intéressant au sujet du contrôle de la raison et de l'imagination chez Supervielle :

Il n'aime guère le plein jour de la conscience, où la raison monte la garde et repousse toutes les suggestions venues du rêve; il n'aime pas non plus le rêve où la conscience disparaît, où les images affluent dans le désordre et dans l'incohérence. La 'rêverie surveillée' lui convient parfaitement, car s'y affleurent sans cesse des lambeaux de rêves ou de fantasmes errants, (...), la conscience demeure vigilante et exerce un certain contrôle (Favre, $1981: 89$ ).

Mais le conteur de l'histoire use également d'une autre subtilité esthétique pour faire partager cet état intermédiaire dans lequel il se trouve : il charge ses détails de souvenirs, d'émotions pour produire « des effets de choc et d'humanisation » (Roy, $1970: 51$ ) chez le lecteur. Ainsi, l'« album de photographies» (Supervielle, 1958: 12), le «grand cartable enfermant des cahiers, une grammaire, une arithmétique, une histoire de France, une géographie " (Supervielle, 1958: 13) sont-ils des objets concrets, issus de la réalité palpable, de la vie antérieure de la fillette, et sont porteurs d'informations terrestres, d'émotions qui semblent la protéger contre ce qu'elle ignore encore. Situé entre la vie et la mort, le personnage s'accroche au matériel, au connu, à ce qui lui parait encore « être dans le vrai» (Supervielle, 1958: 12), et ce sont ces fragments de vie, ces liaisons affectives au terrestre qui éveillent en elle le désespoir de la solitude. Tout contact avec la réalité est néfaste au personnage, dans la mesure où ces parcelles de vie ne sont, en fait, pas la vie et la font sombrer dans la tristesse ; la fillette essaie, constamment, d'être en contact avec la réalité pour se donner un semblant de vie :

Et l'histoire, la géographie, les pays, les grands hommes, les montagnes, les fleuves et les frontières, comment s'expliquer tout cela pour qui n'a que la rue vide d'une petite ville, au plus solitaire de l'Océan. Mais l'Océan même, celui qu'elle voyait sur les cartes, elle ne savait pas se trouver dessus, bien qu'elle l'eût pensé un jour, une seconde. Mais elle avait chassé l'idée comme folle et dangereuse (Supervielle, 1958 : 13).

Le tragique prend, alors, progressivement place au sein de la diégèse : au fur et à mesure que le personnage s'humanise, son désespoir augmente et devient insurmontable. Elle est malheureuse dans ce monde de l'au-delà et songe au monde de l'ici-bas :

- Si j'avais seulement un peu de neige des hautes montagnes la journée passerait plus vite.

- Ecume, écume autour de moi, ne finiras-tu pas par devenir quelque chose de dur.

- Pour faire une ronde il faut au moins être trois. 
- C'étaient deux ombres sans tête qui s'en allaient sur la route poussiéreuse.

- La nuit, le jour, le jour, la nuit, les nuages et les poissons volants (Supervielle, $1958: 14)$ de l'esprit » (Paseyro, 2002 : 175), une illusion poussée à l'extrême et qui déborde dans la vie réelle :

Un jour, lasse de ressembler avec ses nattes et son front très dégagé à la photographie qu'elle regardait dans son album, elle s'irrita contre elle-même et son portrait, et répandit violemment ses cheveux sur ses épaules, espérant que son âge en serait bouleversé (Supervielle, $1958: 15$ ).

En fait, l'enfant mène une triste vie éternelle et songe constamment à la Terre, jusqu'à ce que, fatiguée de sa condition de morte-vivante, elle se révolte. Or, tout reste inchangeable, puisque dans le royaume des ombres on ne peut appréhender le réel. La réalité n'est, en fait, pour un fantôme, qu'illusoire :

Il y avait longtemps que cette vague aurait voulu faire quelque chose pour l'enfant, mais elle ne savait pas quoi.

(...) Après s'être agenouillée devant elle à la manière des vagues, et avec le plus grand respect, elle l'enroula au fond d'elle-même, la garda un très long moment en tâchant de la confisquer, avec la collaboration de la mort. Et la fillette s'empêchait de respirer pour seconder la vague dans son grave projet.

(...) Enfin, voyant que rien n'y faisait, qu'elle ne parviendrait pas à lui donner la mort, la vague ramena l'enfant chez elle dans un immense murmure de larmes et d'excuses.

Et la fillette qui n'avait pas une égratignure dut recommencer d'ouvrir et de fermer les volets sans espoir, et de disparaitre momentanément dans la mer dès que le mât d'un navire pointait à l'horizon (Supervielle, $1958: 17$ ).

En tant que fantôme, l'enfant appartient au monde de l'invisible et du silence et elle ne parvient, par conséquent, pas à entrer dans le domaine de l'oubli. Elle se trouve condamnée à vivre dans l'éternité et sa condition n'est plus semblable à celle des êtres vivants. Elle est sans issue, elle reste prisonnière du néant.

Le lecteur se trouve plongé dans le rêve et le narrateur va se charger de construire le pont qui le lie à la réalité en établissant des analogies entre le monde de l'au-delà et le monde de l'ici-bas :

Marins qui rêvez en haute mer, les coudes appuyés sur la lisse, craignez de penser longtemps dans le noir de la nuit à un visage aimé. Vous risqueriez de donner naissance, dans des lieux essentiellement désertiques, à un être doué de toute la sensibilité humaine et qui ne peut pas vivre ni mourir, ni aimer, et souffre pourtant comme s'il vivait, aimait et se trouvait toujours sur le point de mourir, un être infiniment déshérité dans les solitudes aquatiques, comme cette enfant de l'Océan, née un jour du cerveau de Charles Liévens, de Steenvoorde, matelot de pont du quatre-mâts Le Hardi, qui avait perdu sa fille âgée de douze ans, pendant un de ses voyages et, une nuit, par 55 degrés de latitude Nord et 35 de longitude Ouest, pensa longuement à elle, avec une force terrible, pour le grand malheur de cette enfant (Supervielle, $1958: 18$ ).

Le narrateur clôt son histoire en interpelant les marins et, bien évidemment, tout à la fois, le lecteur, pour leur faire prendre conscience du pouvoir du rêve, de la force de l'esprit sur la matière et de la puissance transfiguratrice de l'imagination. Nous nous sommes abandonnés à l'incantation de la narration, nous nous sommes laissé envoûter par la magie et transporter dans un monde inconnu, mais où le réel a, néanmoins, sa place. Ainsi, lorsque nous découvrons que cette hallucination, que nous avons partagée de 
bon gré avec le narrateur, est empreinte de souvenirs et d'émotions, notre perception change et nous finissons par partager cet état de permanence entre deux mondes. Albert Béguin considère que l'homme doit chercher à s'éloigner du centre du royaume terrestre pour vivre en toute plénitude :

Ici-bas, l'homme «n'existe pas encore ", il est seulement en devenir, et c'est pour cela qu'il est encore « physique et psychique dans l'espace, veillant et dormant dans le temps ». Seuls, des instants insaisissables lui font percevoir, dès maintenant, son unité profonde et deviner ce qu'il y a de passager, de purement terrestre, dans sa dualité. Ces moments sont surtout ceux où, s'éveillant ou s'endormant, il est, pendant une seconde, entre les deux psychés, qui le régissent dans tout le reste de son existence. Sans perdre pourtant la notion de lui-même, il entrevoit là son véritable centre, où âme et corps ne font qu'un (Béguin, 1939 : 96).

Dans ce conte de Supervielle, la tonalité merveilleuse se trouve atténuée par l'humanisation des êtres surnaturels. Le conteur a su transformer les êtres et le monde pour dépasser, selon Sabine Dewulf, «l'apparente clarté du puits de vérité » et " descendre jusqu'en des régions plus obscures, où se manifestait tout en se dissimulant la profondeur d'un univers unique, le monde d'un être individuel " (Dewulf, 2000, TI : 19). Un désir de partage doit donc exister chez le lecteur pour que les barrières conceptuelles puissent être rompues et pour qu'une nouvelle dimension puisse être atteinte. Par le décryptage, un autre monde doit émerger de la superficie du texte, en même temps semblable et différent du nôtre.

"L'Enfant de la haute mer ", de Supervielle, procède à la déréalisation du monde et nous fait glisser vers l'indéfinissable, c'est-à-dire le rêve. L'écrivain a toujours voulu se séparer de la fausse transparence de la réalité et plonger de plein pied dans le mystère de l'univers qui l'entoure, et c'est par ce dilemme intérieur qu'il atteint la magique plénitude de son récit.

\section{BIBLIOGRAPHIE}

BACHELARD, Gaston (1942). L'eau et les rêves. Paris : Corti.

BACHELARD, Gaston (1960). La poétique de la rêverie. Paris : Puf.

BACHELARD, Gaston (1978). La poétique de l'espace. Paris : Puf.

BACHELARD, Gaston (1993). La dialectique de la durée. Paris : Puf.

BEGUIN, Albert (1939). L'âme romantique et le rêve. Paris : Corti.

CROHMÀlNiCEANU, Ovid S. (1995). « Contes d'un poète », Europe, 792, pp. 82-88.

DEWULF, Sabine (2000). Jules Supervielle ou la connaissance poétique : Sous le " soleil de l'oubli », tome I :

Le renoncement au savoir. Paris : L'Harmattan.

DEWULF, Sabine (2000). Jules Supervielle ou la connaissance poétique : Sous le « soleil de l'oubli », tome II :

Une autre connaissance. Paris : L'Harmattan. 
DUPUIS, Michel, MINGELGRÜN, Albert (1987). « Pour une poétique du réalisme magique », in Jean Weisgerber (dir.). Le Réalisme magique : Roman, peinture, cinéma. Bruxelles : L'Age d'Homme, pp. 219-232.

FAVRE, Yves-Alain (1981). Supervielle, la rêverie et le chant dans « Gravitations ». Paris : Nizet.

GROJNOWSKI, Daniel (2000). Lire la nouvelle. Paris : Nathan.

HIDDLESTON, James A. (1965). L'Univers de Jules Supervielle. Paris : Corti.

MENDILOW, Adam Abraham (1952). Times and the novel. Holland: Ysel Press.

PASEYRO, Ricardo (2002). Jules Supervielle - Le forçat volontaire. Paris : Editions du Rocher.

POULET, Georges (1964). Etudes sur le temps humain/3. Paris : Plon.

RoY, Claude (1970). Jules Supervielle. Paris : Seghers.

SUPERVIELLE, Jules (1958). L'Enfant de la haute mer. Paris : Gallimard.

\section{RÉSUMÉS}

Lorsque nous pénétrons dans la mer superviellienne, par le biais de L'Enfant de la haute mer, il nous faut accepter que l'incongru et l'insolite font partie du réel. L'ambiguité de ce monde, tout en même temps proche et lointain du nôtre, nous contraint à redéfinir les frontières entre le réel et le surnaturel, le visible et l'invisible. La rue flottante ou, plus précisément, l'île déserte, est un lieu où le regard se perd, où aucun point fixe n'aide à ordonner et à organiser la vision. Ainsi dépouillée de ses déterminations ordinaires - spatiales et temporelles -, cet élément de la nature constitue une sorte de néant, impossible à exploiter.

In delving into Jules Supervielle's sea, through L'Enfant de la haute mer, we must accept that the incongruous and unusual are part of what is real. The ambiguity of this world, at the same time near and distant from ours, requires us to redefine the boundaries between reality and the supernatural, what is visible and what is invisible. The floating street or, more precisely, the desert island, is a place where one's gaze is lost, where no stable point can help order and organize one's vision. Thus stripped of its usual determinations - spatial and temporal - this element of nature is a kind of néant that is impossible to explore.

\section{INDEX}

Mots-clés : mer, indéfinissable, rêve, Enfant de la haute mer (L'), Supervielle (Jules)

Keywords : sea, undefinable, dream, Enfant de la haute mer ( $\left.\mathrm{L}^{\prime}\right)$, Supervielle (Jules)

\section{AUTEUR}

\section{MARIA EUGÉNIA PEREIRA}

Université de Aveiro

epereira[at]ua.pt 